\title{
Majorana Bound State Leakage to Impurity in Su-Schrieffer-Heeger-Rashba Scenario
}

\author{
A. KobIAŁKA ${ }^{a, *}$ And A. PtOK ${ }^{b}$ \\ ${ }^{a}$ Institute of Physics, Maria Curie-Skłodowska University, \\ Pl. M. Skłodowskiej-Curie 1, 20-031 Lublin, Poland \\ ${ }^{b}$ Institute of Nuclear Physics, Polish Academy of Sciences, \\ W.E. Radzikowskiego 152, 31-342 Kraków, Poland \\ Doi: 10.12693/APhysPolA.138.673 \\ *e-mail: akob@kft.umcs.lublin.pl
}

\begin{abstract}
We show the anomalous features of the Majorana bound state leakage in the situation where topological Rashba nanowire is dimerized according to the $\mathrm{Su}-\mathrm{Sch}$ rieffer-Heeger scenario and an impurity is present at one of the ends of the system. We find that two topological branches: the usual, indigenous to the Rashba nanowire, and the dimerized one, existing as a result of the Su-Schrieffer-Heeger dimerization of the nanowire, have different asymmetry of spin polarization that can be explained by the opposite order of bands taking part in topological transitions. Additionally, the introduction of an impurity to the dimerized nanowire influences the leakage of the Majorana bound states into the trivial impurity due to the emergence of the Andreev bound states that behave differently depending on whether the system is or is not in a topological phase. This results in the pinning of zero energy states to the impurity site for some range of parameters.
\end{abstract}

topics: Su-Schrieffer-Heeger, SSH, Rashba nanowire, Majorana bound states

\section{Introduction}

Systems exhibiting an existence of the Majorana bound states (MBS) are very promising for the emergence of a new branch of quantum computing - topological quantum computing, relying on topological superconductors. Quantum computing is a steadily growing field of both physics and nanotechnology, however, a working example of its topological counterpart is still yet to be presented. A presumed advantage of topological quantum computing over a "regular" one is the property of faulttolerant computing [1]. In order to achieve this, non-Abelian quasiparticles [2] have to be employed, hence the interest in MBS which are believed to possess such properties [3].

Recently, such quasiparticles have been experimentally uncovered in numerous examples, both in one-dimensional (1D) systems (e.g., in the form of zero-energy bound states localized at the ends of nanowires deposited upon a surface due to interplay between spin-orbit coupling, superconductivity and magnetic field) [4-15] or two-dimensional (2D) systems (e.g., edge states around a superconducting island) [16-18].

Dimerization alone can allow for a topological transition, even if a superconductor is not present in the system [19]. For instance, in the Su-SchriefferHeeger (SSH) model [19, 20], two different bonds between atoms are assumed which makes the atoms dimerize due to the Peierls instability. This phenomenon generated some interest but mainly the combination of the Kitaev [2] and SSH models [21-27] was used. Therefore, we combine the aforementioned SSH dimerization with the Rashba nanowire properties in order to obtain a Su-Schrieffer-Heeger-Rashba (SSHR) model.

MBS, as the edge phenomena, tend to leak to the furthest elements of the system, even if those parts (e.g., impurity) do not manifest any topologically non-trivial nature [28-31]. At this point, we check how the leakage of MBS behaves when impurity is attached to the end of the dimerized Rashba nanowire, within the SSH scenario (see Fig. 1), depending on the order of the bond strength and thus the type of the bond between the last two sites in the system.

This paper is organized as follows: in Sect. 2 the SSH model of the dimerized Rashba nanowire and methods is introduced, in Sect. 3 results obtained by numerical calculation are discussed and in Sect. 4 the results are summerized.

\section{Methodology}

We consider an SSH analogue of the Rashba nanowire, where the 1D semiconducting nanowire which is deposited on a superconducting substrate (Fig. 1) is modified with an alternating order of weak and strong bonds (or vice versa) that emulate 


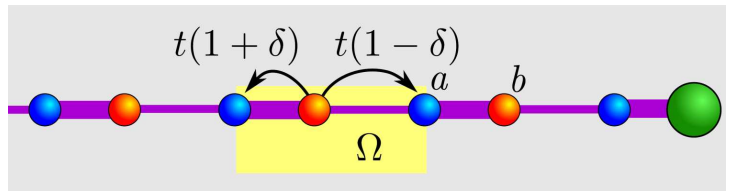

Fig. 1. Schematics of the dimerized nanowire proximitized to the isotropic superconductor. Modulation of the hopping integral $\delta$ corresponds to the shifts in positions between the neighboring $a$ and $b$ sites in the unit cell $\Omega$ (marked by the yellow frame). Sites on selected sublattices $a$ and $b$ are marked by blue and red colors, respectively, while green corresponds to an additional impurity site connected to the end of the nanowire.

an SSH scenario. We model the distance between the neighboring sites $a, b$ (forming the unit cell $\Omega$ ) by the modulation $\delta$ of the hopping integral that effectively changes the probability of electron transport between the neighboring sites. Similar modulations also affect the spin-orbit Rashba interactions. In a natural way, the SSH model describes a system with two sublattices (sites of $a$ - and $b$-type).

\subsection{Microscopic model}

Our system can be described by the Hamiltonian $\mathcal{H}=\mathcal{H}_{0}+\mathcal{H}_{\text {so }}+\mathcal{H}_{\text {prox }}$. The first term

$$
\begin{aligned}
\mathcal{H}_{0} & = \\
& -\sum_{i \sigma}\left[t(1+\delta) c_{i a \sigma}^{\dagger} c_{i b \sigma}+t(1-\delta) c_{i a \sigma}^{\dagger} c_{i-1 b \sigma}+\text { h.c. }\right] \\
& -\sum_{s \in \Omega} \sum_{i, \sigma}(\mu+\sigma h) c_{i s \sigma}^{\dagger} c_{i s \sigma},
\end{aligned}
$$

describes an SSH-like nanowire. The operator $c_{i s \sigma}^{\dagger}$ $\left(c_{i s \sigma}\right)$ denotes the creation (annihilation) of the electron with spin $\sigma$ in $i$-th unit cell and sublattice $s$ (e.g., site $a$ or $b$ ), $\mu$ is the chemical potential, $h$ denotes the magnetic field in the Zeeman form and $(1 \pm \delta)$ is a periodic variation of hopping integral $t$ between the nearest neighboring sites, i.e., between sites in different sublattices. We also assume similar modulation for the spin-orbit Rashba interaction term

$$
\begin{gathered}
\mathcal{H}_{\mathrm{so}}=-i \sum_{i \sigma \sigma^{\prime}}\left[\lambda(1+\delta) c_{i a \sigma}^{\dagger}\left(\sigma_{y}\right)_{\sigma \sigma^{\prime}} c_{i b \sigma^{\prime}}\right. \\
\left.+\lambda(1-\delta) c_{i a \sigma}^{\dagger}\left(\sigma_{y}\right)_{\sigma \sigma^{\prime}} c_{i-1 b \sigma^{\prime}}\right]+ \text { h.c. }
\end{gathered}
$$

where $\sigma_{y}$ is the second Pauli matrix and $\lambda$ describes the strength of the spin-orbit coupling. The last term models a BCS-like superconducting gap that arises from the proximity effect, i.e., the deposition of a nanowire on a superconducting surface [32]:

$$
\mathcal{H}_{\text {prox }}=\sum_{i s}\left(\Delta c_{i s \uparrow}^{\dagger} c_{i s \downarrow}^{\dagger}+\Delta^{*} c_{i s \downarrow} c_{i s \uparrow}\right) .
$$

Impurity is treated as an additional site connected to the nanowire that is not affected by the proximity effect $\Delta_{\text {imp }}=0$.
In a typical situation of a homogeneous nanowire, the transition from the trivial to non-trivial topological phase occurs for some critical value of magnetic field [33-35]:

$$
h_{c}^{2}=(2 t-\mu)^{2}+|\Delta|^{2} .
$$

With the increase of magnetic field, the quasiparticle spectrum closes and reopens as a new topologically non-trivial gap at $h=h_{c}$ [36]. In the case of the dimerized SSH nanowire, the emergence of a non-trivial phase depends on the existence of additional parameters (e.g., $\lambda$ and $\delta$ ). Then, the value of $h_{c}$ depends on model parameters in a non-trivial manner and can be determined analytically (more details can be found in [37]) but still, in the limit of $\delta \rightarrow 0$, the condition (4) remains unchanged.

\subsection{Formalism}

The model Hamiltonian $\mathcal{H}$ can be numerically diagonalized by the Bogoliubov-Valatin transformation [38]:

$$
c_{i s \sigma}=\sum_{n}\left(u_{i s n \sigma} \gamma_{n}-\sigma v_{i s n \bar{\sigma}}^{*} \gamma_{n}^{\dagger}\right) \text {, }
$$

where $\gamma_{n}$ and $\gamma_{n}^{\dagger}$ are the "new" quasiparticle fermionic operators. This transformation yields the Bogoliubov-de Gennes equations, i.e., $\mathcal{E}_{n} \Psi_{i s n}=$ $\sum_{j s^{\prime}} \mathbb{H}_{i s, j s^{\prime}} \Psi_{j s^{\prime} n}$, where the Hamiltonian $\mathbb{H}_{i s, j s^{\prime}}$ is given in the matrix form as

$$
\begin{aligned}
& \mathbb{H}_{i s, j s^{\prime}}= \\
& \left(\begin{array}{cccc}
H_{i s, j s^{\prime}, \uparrow} & D_{i s, j s^{\prime}} & S_{i s, j s^{\prime}}^{\uparrow \downarrow} & 0 \\
D_{i s, j s^{\prime}}^{*} & -H_{i s, j s^{\prime}, \downarrow}^{*} & 0 & S_{i s, j s^{\prime}}^{\downarrow \uparrow} \\
S_{i s, j s^{\prime}}^{\downarrow \uparrow} & 0 & H_{i s, j s^{\prime}, \downarrow} & D_{i s, j s^{\prime}} \\
0 & S_{i s, j s^{\prime}}^{\uparrow \downarrow} & D_{i s, j s^{\prime}}^{*} & -H_{i s, j s^{\prime}, \uparrow}^{*}
\end{array}\right)
\end{aligned}
$$

while eigenvector

$$
\Psi_{i s n}=\left(u_{i s n \uparrow}, v_{i s n \downarrow}, u_{i s n \downarrow}, v_{i s n \uparrow}\right)^{T} .
$$

The matrix block elements (taking into account both sublattices) are given here by

$$
\begin{aligned}
& H_{i s, j s^{\prime}, \sigma}=-t(1+\delta) \delta_{i j} \delta_{\left\langle s s^{\prime}\right\rangle} \\
& \quad-t(1-\delta) \delta_{i-1, j} \delta_{\left\langle s, s^{\prime}\right\rangle}-(\mu+\sigma h) \delta_{i j} \delta_{s s^{\prime}} .
\end{aligned}
$$

In turn, the on-site superconducting gap is denoted as $D_{i s, j s^{\prime}}=\Delta \delta_{i j} \delta_{s s^{\prime}}$, while

$$
\begin{aligned}
& S_{i s, j s^{\prime}}^{\sigma \sigma^{\prime}}=-\mathrm{i} \lambda\left(\sigma_{y}\right)_{\sigma \sigma^{\prime}} \\
& \quad \times\left[(1+\delta) \delta_{i j} \delta_{\left\langle s s^{\prime}\right\rangle}-(1-\delta) \delta_{i-1, j} \delta_{\left\langle s s^{\prime}\right\rangle}\right]
\end{aligned}
$$

stands for the spin-orbit Rashba term. Now, we must keep in mind that the indexes $i$ and $s$ change values over a number of unit cells and sublattice indexes, respectively. From this, $\mathbb{H}_{i s, j s^{\prime}}$ is a square matrix with the size of $4 \mathcal{N} \times 4 \mathcal{N}$, where $\mathcal{N}$ denotes a number of sites in the system. In the absence of impurity, $\mathcal{N}$ is equal to the double of cells number $N_{\Omega}$.

From the solution of the BdG equations, we can determine the spin-resolved local density of states 
$(\operatorname{LDOS}) \rho_{i s \sigma}(\omega)=-\frac{1}{\pi} \operatorname{Im}\left\langle\left\langle c_{i s \sigma} \mid c_{i s \sigma}^{\dagger}\right\rangle\right\rangle$ which can be expressed as [39]:

$$
\begin{aligned}
& \rho_{i s \sigma}(\omega)=\sum_{n}\left|u_{i s n \sigma}\right|^{2} \delta\left(\omega-\mathcal{E}_{n}\right) \\
& \quad+\left|v_{i s n \sigma}\right|^{2} \delta\left(\omega+\mathcal{E}_{n}\right)
\end{aligned}
$$

Also, the spin polarization asymmetry (SPA) of LDOS

$$
\delta \rho_{i s}(\omega)=\rho_{i s \uparrow}(\omega)-\rho_{i s \downarrow}(\omega)
$$

can give additional information, e.g., about spin polarization of the bound state [40]. In numerical calculations, we replace the Dirac delta function by Lorentzian $\delta(\omega)=\zeta /\left[\pi\left(\omega^{2}+\zeta^{2}\right)\right]$ with a small broadening $\zeta / t=0.001$.

Total LDOS $\rho_{i s \uparrow}(\omega)+\rho_{i s \downarrow}(\omega)$ in a low temperature limit gives information about the differential conductance $G(\omega)$ [41-43]. Similarly, SPA LDOS $\delta \rho_{\text {is }}$ can give information about spin polarization of the bound states. Both quantities can be measured in a relatively simple way by using a scanning tunneling microscope (STM) [44-46]. Experiments with a magnetic tip give information about the magnetic structure of the bound states in atomic scale [47-49]. From the theoretical point of view, previous studies in spinfull models have shown that MBS have spin polarization $[28,29,40,50]$. From this, an existence of topological bound states can be probed via the previously mentioned spin-polarized STM measurements [44-46] (which has been done, e.g., in ferromagnetic atom chains $[10,11])$. This type of measurements can be useful in distinguishing between the ordinary Andreev bound states (ABS) and topological MBS in hybrid nanostructures [51].

A similar analysis of the system can be performed in the momentum space (more details are given in [37]). The studies are based on the spin-resolved spectral function

$$
\mathcal{A}_{\boldsymbol{k} \sigma}(\omega)=-\frac{1}{\pi} \operatorname{Im}\left\langle\left\langle c_{\boldsymbol{k} \sigma} \mid c_{\boldsymbol{k} \sigma}^{\dagger}\right\rangle\right\rangle,
$$

from which the band structure and its SPA $\delta \mathcal{A}_{\boldsymbol{k}}(\omega)=\mathcal{A}_{\boldsymbol{k} \uparrow}(\omega)-\mathcal{A}_{\boldsymbol{k} \downarrow}(\omega)$ can be found [52]. Similarly to LDOS, these quantities can be measured via the angle-resolved photoemission spectroscopy (ARPES) technique [53], even in nanostructures [54]. The existence of the topological phase in the system leads to the observation of the band inversion, clearly visible in the spin polarization of bands. This is typical not only of the case of the topological insulator $[52,55]$ but also of other systems in which the topological phase emerges [29, 56, 57].

\section{Numerical results}

In this section, we discuss the leakage of MBS to the impurity within the dimerized SSH nanowire. As for the parameters used in calculation, we took a nanowire composed of $N_{\Omega}=100$ cells, i.e., $\mathcal{N}=200$ sites and an additional impurity being the 201st site (unless stated otherwise). An alternating order of bonds is preserved in the junction between the nanowire and impurity. Nanowire is characterized by $\Delta / t=0.2$ and $\lambda / t=0.15$. Any change in chemical potential $\mu$ affects the entire system, both the nanowire and impurity. At this point, it should be mentioned that the described results do not depend on the size of the nanowire. Additionally, throughout the entire paper, we take $h=0.3 t>h_{c}$ which ensures that the homogeneous system is in the non-trivial phase. If not stated differently, when the nanowire has an odd number of sites, it begins with a weak $(1-\delta) t$ bond and ends with a strong $(1+\delta) t$ bond.

The existence of hopping modulation has a negative impact on the usual non-trivial phase. However, for the dimerization-dependent branch it is essential for its existence. Let us start with discussing the influence of the impurity on the Rashba nanowire.

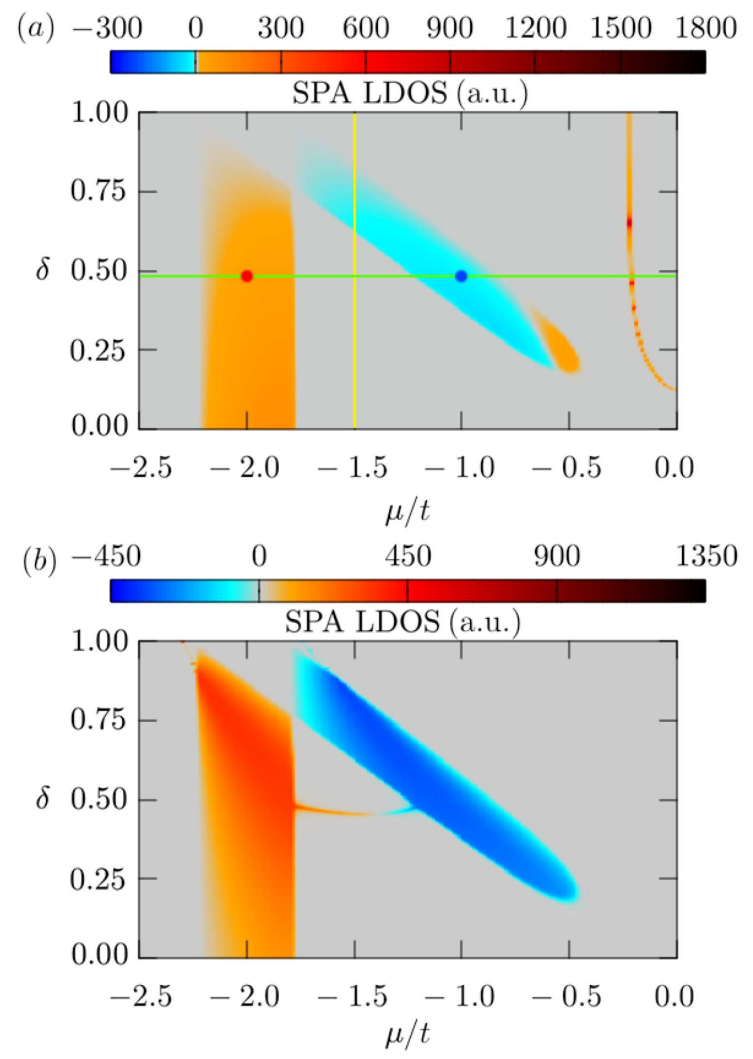

Fig. 2. SPA LDOS for zero energy $\mu-\delta$ phase space of the first site (a) of the system and impurity site (b). The first site is connected to the rest of the nanowire with a weak bond, while the impurity site is connected by a strong bond. In the case of (b), the impurity is connected with a strong bond, which allows for forming of the bridge-like structure. Eigenvalues for parameters along the green and yellow lines at (a) are shown in Fig. 3. Red and blue dots correspond to SPA $\delta \mathcal{A}_{\boldsymbol{k}}(\omega)$ for different topological phases (cf. Fig. 5a and 5b respectively). Results for the system with $\mathcal{N}=201$ sites and $h / t=0.3$. 

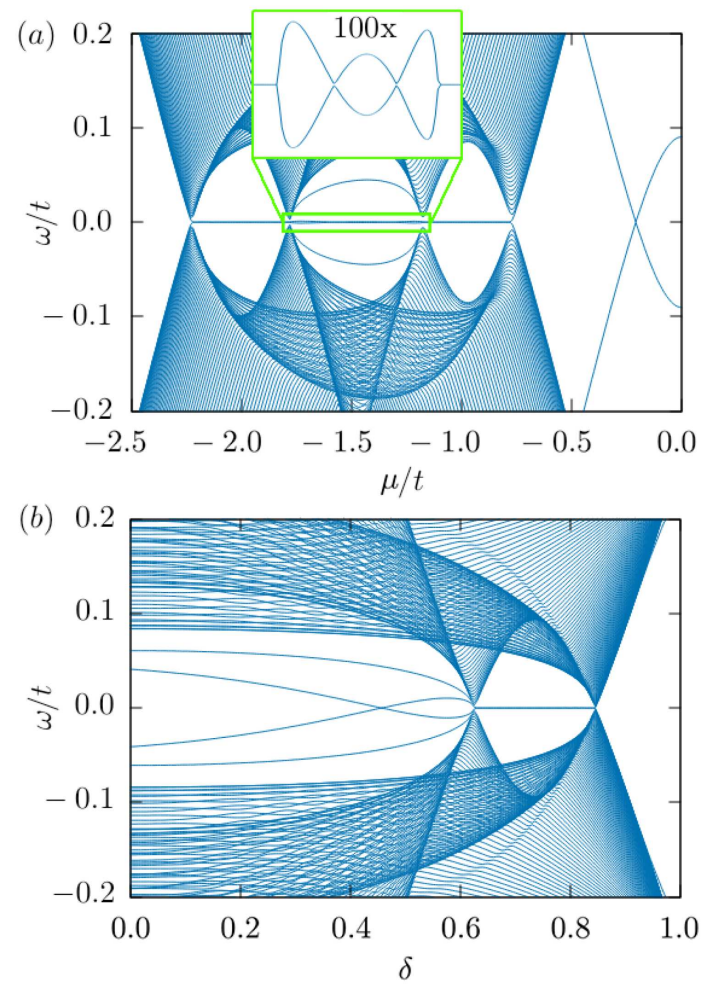

Fig. 3. Spectrum of the system for fixed $\delta=0.46$ (a) and $\mu / t=-1.5$ (b) which corresponds to the green horizontal and yellow vertical lines in Fig. 2a, respectively. Results in the absence of impurity are presented in Fig. 8 in [37].

In Fig. 2, we show a color map of SPA LDOS for $\omega=0$ as a function of the chemical potential $\mu$ and hopping modulation $\delta$. The nanowire is in the presence of the impurity which is connected to the system with a strong bond $(1+\delta)$. In Fig. 2, regions centered around $\mu=2 t$ (near the bottom of the band) show parameters of the system which allow for hosting of MBS in the system. This range of parameters, where the non-trivial phase exists, can be associated with a typical limit in the homogeneous system [33-35]. Additional modulation of hopping introduced by $\delta$ does not change the topological character of the system in $\delta \rightarrow 0$. However, bond modulation creates an additional topological branch which allows for the existence of MBS in a broader range of parameters, in accordance with (4). This additional dimerized branch incorporates regions within the band where for some range of modulation of hopping integrals a nontrivial phase appears in which MBS can emerge. The abrupt change of SPA of the system between two branches of a topological phase can be explained by the reordering of bands that takes place with each band closure at the moment of a topological transition [58]. When the bands close at the transition from a topologically non-trivial to a trivial state $\mu \simeq 1.8 t$, they reopen in the opposite order during the transition to a non-trivial state (within a dimerized topological branch) [58].
Due to the interplay between the magnetic field and SOC, the change of spin polarization occurs. In Fig. 2a, we can see a phase space for the first site of the nanowire, linked to the main part of the nanowire with a weak bond $(1-\delta)$. This allows for visualization of a characteristic feature for the investigated system, a parabola at $\mu / t \in(-0.22,0.22)$ (as plots are $\mu$-symmetric) which is a manifestation of states of the first site of the nanowire, crossing at zero energy. Another distinctive feature is shown in Fig. 2b, where we can see a SPA LDOS space for the impurity (being the last site of the nanowire) which is linked to the main part of the nanowire with a strong bond $(1+\delta)$.

The existence of bridge-like features can be understood from the analysis of the system spectrum presented in Fig. 3. There, a bridge-like feature emerges due to the existence of ABS, connecting separate topological phases. It is the result of crossing the Fermi level by the eigenvalues of states associated with the existence of impurity, coupled to the nanowire by a strong bond. There is no analogue of strong bond feature for the last site when it is not an impurity. In Fig. 3a, we can see eigenvalues for $\delta=0.46$ (green line in Fig. 2a), crossing the bridge-like structure. Here, the two zero energy Majorana states are separated by a trivial bow tielike ABS feature (inset). These in-gap states are also clearly visible in the SPA LDOS analyses and are strongly associated with the localization of ABS from one site of the nanowire - near the impurity, as seen in Fig. 4. As it may be observed, this structure is in fact a manifestation of zero energy crossing of ABS. Similar behavior can be observed in the case of the spectrum of the system from $\mu / t=-1.5$ (yellow line in Fig. 2a), shown in Fig. 3b.

In contrast to MBS in an isotropic chain $(\delta=0)$, in our results SPA LDOS of MBS have the opposite value in a different part of the phase space (Fig. 2). This behavior is strongly associated with the influence of $\delta$ on the band structure and its spin polarization (Fig. 5). The exact analysis of the band structure where MBS exist [29] shows that the Majorana quasiparticle inherits spin polarization of bands nearest the zero energy, i.e., the Fermi level. Here, from studying the band structure, we can observe that MBS in the main branch have a typical spin polarization $\uparrow$ (Fig. 5a). In this case, the emergence of the topological phase is associated with the band inversion around $k=0$. On the contrary, SPA of MBS in dimerization-dependent branch is $\downarrow$, namely the opposite. This is a consequence of the band inversion of the nearly fully filled bands around $k=\pi$ point (Fig. 5b). Summarizing, in our case SPA LDOS yielded unexpected results if compared to the aforementioned results.

The crossing point shows accidental nature of a bridge-like feature of zero energy ABS. Thanks to this, it is certain that the region connecting two topological branches does not hold MBS, as this would result not only in a zero energy state typical 

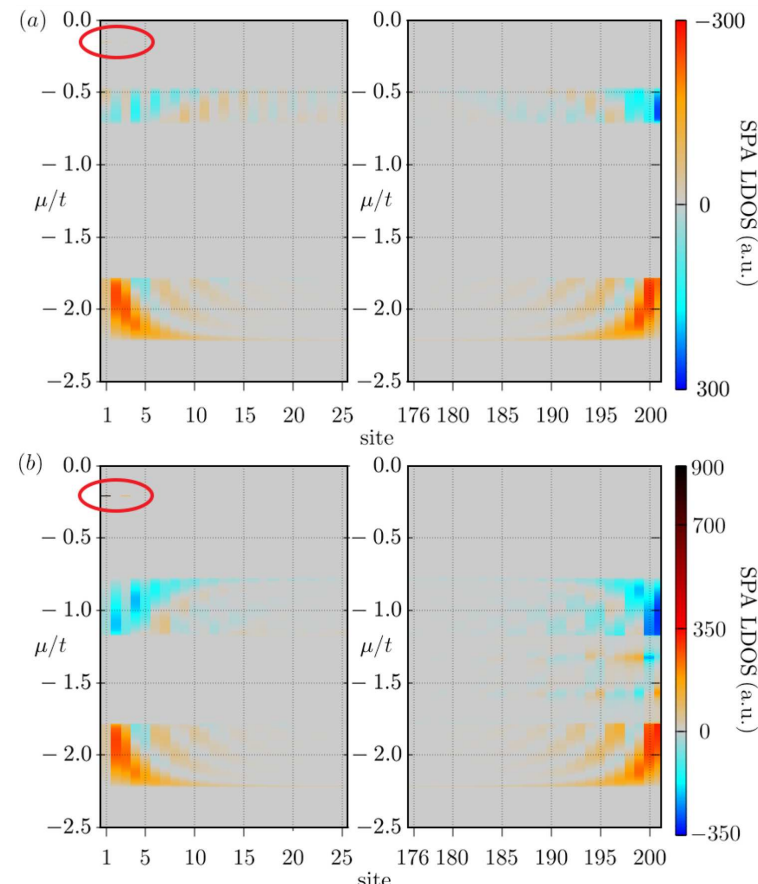

Fig. 4. Real space distribution of asymmetry of SPA of MBS as a function of the chemical potential, at the ends of the nanowire. Results for parameters like in Fig. 2 along $\delta=0.24$ (a) and $\delta=0.46$ (b). The central feature for $\mu / t \simeq-1.5$ shows the distribution of SPA LDOS along the bridge-like structure from Fig. 2. Regions within the red oval show instances of a weak bond parabola state forming on the first site. Here, our system consists of $\mathcal{N}=200$ sites.

of MBS but additionally with an avoided crossing of ABS. On the other hand, if the nanowire is pristine (no impurity), near-zero energy states that do not mutate into MBS after the topological transition would not show any avoided crossing or bow tie behavior but instead they will follow MBS and diverge out of the topological regime.

Now, we discuss the zero-energy SPA LDOS shown in Fig. 4. In the case of a non-trivial phase, MBS are localized at both ends of the nanowire. These states are characterized by the oscillation of SPA LDOS in space. As we can see, in both branches of the non-trivial phase LDOS is characterized by the opposite SPA. The largest localization of the state is visible at the impurity site (right-hand side), i.e., $\mu / t \approx-2$ for the main branch and $\mu / t \approx-0.75$ for the dimerized branch, while ABS are pinned to the impurity. For the intermediate region $\mu / t \approx-1.5$, we observe the localization of the state mostly at impurity, which is associated with the aforementioned ABS that were manifested as a bridge-like structure in the phase space and, correspondingly, a bow tie region in eigenvalues of Fig. 3. As we move away from the impurity towards the middle of the nanowire, a bridge-like feature will fade away and show no SPA within

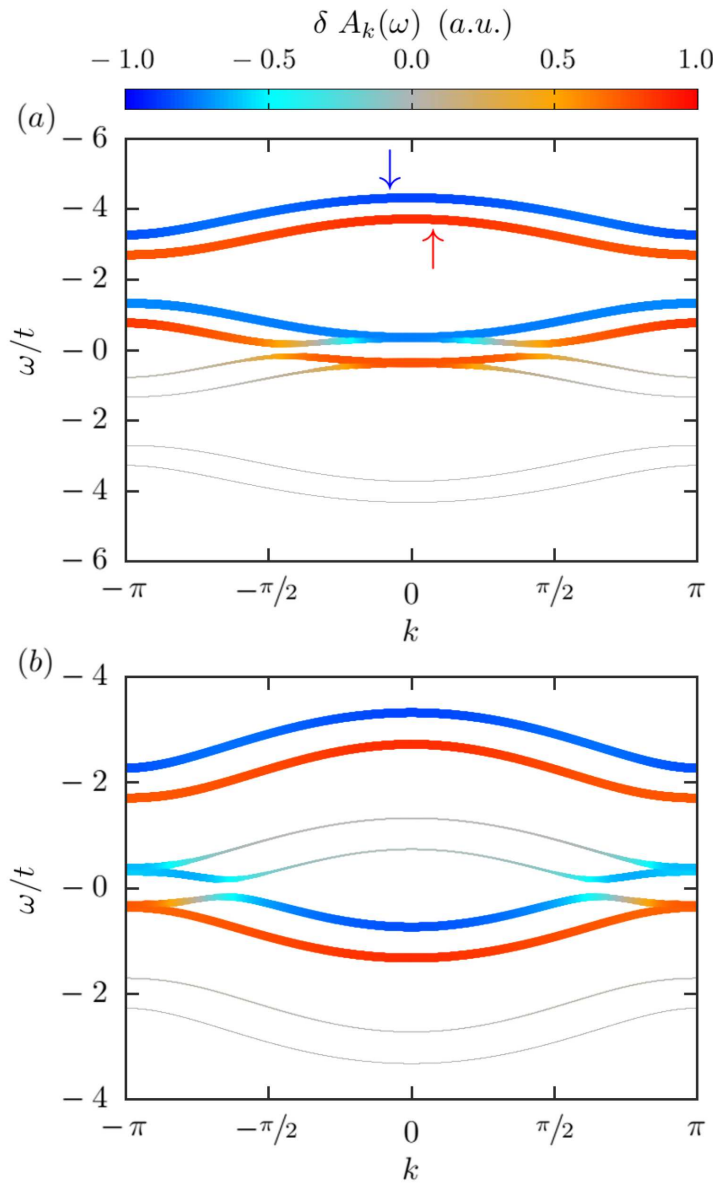

Fig. 5. SPA of the spectral function $\delta \mathcal{A}_{k}(\omega)$. Results for (a) $\mu / t=-2$ and (b) $\mu / t=-1$, with fixed $\delta=0.46$ (see red and blue dots in Fig. 2a, respectively). The color corresponds to spin polarization (marked with corresponding arrows) and the width of line to the total spectral function $\mathcal{A}_{\boldsymbol{k} \uparrow}(\omega)+\mathcal{A}_{\boldsymbol{k} \downarrow}(\omega)$.

a distance of $\approx 20$ sites. Additionally, we can observe instances of weak bond parabola states forming on the first site (red ovals). These states are characterized by high SPA LDOS and correspond to ABS forming on the edge site which is weakly connected to the rest of the nanowire.

We should also discuss an important problem of interplay between trivial energy levels (of quantum dot or impurity) with energy levels of SSHR chain which contains MBS in a topological regime. In a typical case, when additional impurity is connecting to the trivial superconducting system, the ordinary in-gap Andreev bound states emerge [59]. The situation is more interesting when impurity is connected to the superconducting system in a topological phase. For instance, this issue was experimentally studied by Deng et al. [60], in a fabricated nanowire with a quantum dot at one end. Topologically trivial bound states were seen to coalesce into MBS as the magnetic field was increased. A theoretical study of this behavior showed that the interplay between trivial ABS and 

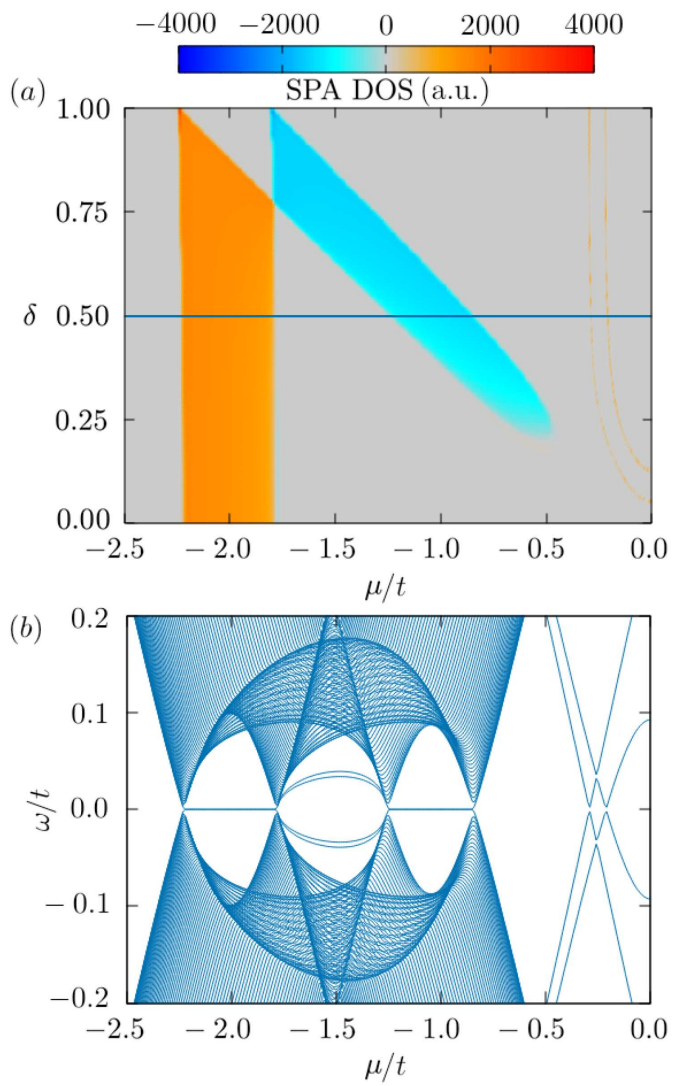

Fig. 6. (a) SPA for zero energy DOS phase spaces as a function of $\mu$ and $\delta$, in the case when the nanowire begins with a weak bond and connects with a weak bond to impurity (as seen in Fig. 2). Here, our system consists of $\mathcal{N}=200$ sites. (b) Eigenvalues for the cross-section of (a), along the blue line $(\delta=0.5$, as seen in Fig. 3).

topological MBS strongly depend on spin polarization of ABS [28, 56, 61, 62], due to positive spin polarization of MBS [29]. In such a case, the avoided crossing or resonance of the ABS energy levels can be observed [63-65]. Moreover, this behavior can be helpful in distinguishing MBS from ABS [66-68]. At this point, we must have in mind that the boundary of the topological regime of a one-dimensional nanowire is given by relation (4) $[56,69]$. In this regime, MBS have the same spin polarization [29]. Contrary to this, in the discussed SSHR model, a topological phase diagram has a more complicated form - due to the existence of the main and dimerization-dependent branches (see Fig. 3) [37]. Here, the spin polarization of MBS depends on parameters of the system, i.e., in the main (dimerized) branch it is positive (negative). Unfortunately, this can lead to ambiguity in distinguishing between ABS and MBS.

Finally, we analyze the results for the system with the even (200 in total) number of sites. In such a case, the nanowire begins with a weak bond and connects with a weak bond to impurity (Fig. 6). The number of sites does not affect the results in any other way than just the order of weak/strong bonds. Here, we can see a familiar phase space with two additional parabolas in zero-energy SPA LDOS (Fig. 6a) forming at $\mu<0.3 t$. The $\mu$-position of the starting point for the outer parabola is linearly dependent on the value of the magnetic field. As for the inner parabola, it forms only if the system exists in a non-trivial phase, after the gap closing $\left(h>h_{c}\right)$, similar to the bridge-like feature. If the nanowire started and ended with a strong bond, a bridge-like feature identical to the one from Fig. 2b would appear. However, the fact of both bonds being the same would not affect the bridge in any way, in contrast to the situation with a weak bond.

\section{Summary}

In this paper, we have shown that the Majorana bound state leakage in the Rashba nanowire which is dimerized according to the SSH scenario might behave anomalously, when an additional impurity is in the vicinity of the nanowire. We find that topological branches, the usual and the dimerized ones, have different SPA that can be explained by the opposite order of bands taking part in topological transitions which are closest to the Fermi level. Moreover, the introduction of impurity along the dimerized nanowire influences the leakage profile of the Majorana state into the trivial impurity. Coupling of impurity to the nanowire leads to the emergence of the trivial Andreev bound states, strongly localized around the impurity. In the case of the one-site impurity, this can lead to the emergence of states crossing the Fermi level. As a consequence, we observe trivial zero-energy states in the form of a bridge-like structure, connecting two branches of the non-trivial topological phases. Stemming from this, measurements of both ends of the nanowire in search of MBS could resolve an ambiguity created by a potential existence of impurities in the nanowire.

\section{Acknowledgments}

We thank Pascal Simon and Nicholas Sedlmayr for inspiring discussions. This work was supported by the National Science Centre (NCN, Poland) under grants UMO-2031/N/ST3/01746 (A.K.) and UMO-2017/25/B/ST3/02586 (A.P.).

\section{References}

[1] D. Aasen, M. Hell, R.V. Mishmash et al., Phys. Rev. X 6, 031016 (2016).

[2] A.Y. Kitaev, Phys.-Usp. 44, 131 (2001).

[3] Ch. Nayak, S.H. Simon, A. Stern, M. Freedman, S. Das Sarma, Rev. Mod. Phys. 80, 1083 (2008). 
[4] V. Mourik, K. Zuo, S.M. Frolov, S.R. Plissard, E.P.A.M. Bakkers, L.P. Kouwenhoven, Science 336, 1003 (2012).

[5] A. Das, Y. Ronen, Y. Most, Y. Oreg, M. Heiblum, H. Shtrikman, Nat. Phys. 8, 887 (2012).

[6] S. Nadj-Perge, I.K. Drozdov, J. Li, H. Chen, S. Jeon, J. Seo, A.H. MacDonald, B.A. Bernevig, A. Yazdani, Science 346, 602 (2014).

[7] R. Pawlak, M. Kisiel, J. Klinovaja, T. Meier, S. Kawai, T. Glatzel, D. Loss, E. Meyer, Npj Quant. Informat. 2, 16035 (2016).

[8] M.T. Deng, S. Vaitiekenas, E.B. Hansen, J. Danon, M. Leijnse, K. Flensberg, J. Nygård, P. Krogstrup, C.M. Marcus, Science 354, 1557 (2016).

[9] F. Nichele, A.C.C. Drachmann, A.M. Whiticar, Phys. Rev. Lett. 119, 136803 (2017).

[10] S. Jeon, Y. Xie, J. Li, Z. Wang, B.A. Bernevig, A. Yazdani, Science 358, 772 (2017).

[11] H. Kim, A. Palacio-Morales, T. Posske, L. Rózsa, K. Palotás, L. Szunyogh, M. Thorwart, R. Wiesendanger, Sci. Adv. 4, aar5251 (2018).

[12] R.M. Lutchyn, E.P.A.M. Bakkers, L.P. Kouwenhoven, P. Krogstrup, C.M. Marcus, Y. Oreg, Nat. Rev. Mater. 3, 52 (2018).

[13] Ö. Gül, H. Zhang, J.D.S. Bommer, Nat. Nanotechnol. 13, 192 (2018).

[14] A. Fornieri, A.M. Whiticar, F. Setiawan et al., Nature 569, 89 (2019).

[15] H. Ren, F. Pientka, S. Hart et al., Nature 569, 93 (2019).

[16] G.C. Ménard, S. Guissart, Ch. Brun et al., Nat. Commun. 8, 2040 (2017).

[17] G.C. Ménard, A. Mesaros, Ch. Brun, F. Debontridder, D. Roditchev, P. Simon, T. Cren, Nat. Commun. 10, 2587 (2019).

[18] A. Palacio-Morales, E. Mascot, S. Cocklin, H. Kim, S. Rachel, D.K. Morr, R. Wiesendanger, Sci. Adv. 5, eaav6600 (2019).

[19] W.P. Su, J.R. Schrieffer, A.J. Heeger, Phys. Rev. Lett. 42, 1698 (1979).

[20] A.J. Heeger, S. Kivelson, J.R. Schrieffer, W.P. Su, Rev. Mod. Phys. 60, 781 (1988).

[21] R. Wakatsuki, M. Ezawa, Y. Tanaka, N. Nagaosa, Phys. Rev. B 90, 014505 (2014).

[22] Y. Wang, J.-J. Miao, H.-K. Jin, S. Chen, Phys. Rev. B 96, 205428 (2017).

[23] M. Ezawa, Phys. Rev. B 96, 121105 (2017).
[24] G.Y. Chitov, Phys. Rev. B 97, 085131 (2018).

[25] W.C. Yu, P.D. Sacramento, Y.C. Li, D.G. Angelakis, H.-Q. Lin, Phys. Rev. B 99, 115113 (2019).

[26] Ch.-B. Hua, R. Chen, D.-H. Xu, B. Zhou, Phys. Rev. B 100, 205302 (2019).

[27] S. Tamura, S. Nakosai, A.M. BlackSchaffer, Y. Tanaka, J. Cayao, Phys. Rev. B 101, 214507 (2020).

[28] A. Ptok, A. Kobiałka, T. Domański, Phys. Rev. B 96, 195430 (2017).

[29] A. Kobiałka, A. Ptok, J. Phys. Condens. Matter 31, 185302 (2019).

[30] A. Kobiałka, A. Ptok, Acta Phys. Pol. A 135, 64 (2019).

[31] A. Kobiałka, T. Domański, A. Ptok, Sci. Rep. 9, 12933 (2019).

[32] W. Chang, S.M. Albrecht, T.S. Jespersen, F. Kuemmeth, P. Krogstrup, J. Nygård, C.M. Marcus, Nat. Nanotechnol. 10, 232 (2015).

[33] M. Sato, S. Fujimoto, Phys. Rev. B 79, 094504 (2009).

[34] M. Sato, Y. Takahashi, S. Fujimoto, Phys. Rev. Lett. 103, 020401 (2009).

[35] M. Sato, Y. Takahashi, S. Fujimoto, Phys. Rev. B 82, 134521 (2010).

[36] J.E. Moore, L. Balents, Phys. Rev. B 75, 121306 (2007).

[37] A. Kobiałka, N. Sedlmayr, M.M. Maśka, T. Domański, Phys. Rev. B 101, 085402 (2020).

[38] P.G. de Gennes, Superconductivity of Metals and Alloys, Addison-Wesley, 1989.

[39] H. Matsui, T. Sato, T. Takahashi, S.-C. Wang, H.-B. Yang, H. Ding, T. Fujii, T. Watanabe, A. Matsuda, Phys. Rev. Lett. 90, 217002 (2003).

[40] D. Sticlet, C. Bena, P. Simon, Phys. Rev. Lett. 108, 096802 (2012).

[41] J. Figgins, D.K. Morr, Phys. Rev. Lett. 104, 187202 (2010).

[42] D. Chevallier, J. Klinovaja, Phys. Rev. B 94, 035417 (2016).

[43] J. Stenger, T.D. Stanescu, Phys. Rev. B 96, 214516 (2017).

[44] W.A. Hofer, A.S. Foster, A.L. Shluger, Rev. Mod. Phys. 75, 1287 (2003).

[45] R. Wiesendanger, Rev. Mod. Phys. 81, 1495 (2009).

[46] H. Oka, O.O. Brovko, M. Corbetta, V.S. Stepanyuk, D. Sander, J. Kirschner, Rev. Mod. Phys. 86, 1127 (2014).

[47] F. Meier, Lihui Z., J. Wiebe, R. Wiesendanger, Science 320, 82 (2008). 
[48] S.M. Hus, X.-G. Zhang, G.D. Nguyen, W. Ko, A.P. Baddorf, Y.P. Chen, A.-P. Li, Phys. Rev. Lett. 119, 137202 (2017).

[49] S. Rolf-Pissarczyk, S. Yan, L. Malavolti, J.A.J. Burgess, G. McMurtrie, S. Loth, Phys. Rev. Lett. 119, 217201 (2017).

[50] M.M. Maśka, T. Domański, Sci. Rep. 7, 16193 (2017).

[51] P. Devillard, D. Chevallier, M. Albert, Phys. Rev. B 96, 115413 (2017).

[52] A. Bansil, H. Lin, T. Das, Rev. Mod. Phys. 88, 021004 (2016).

[53] A. Damascelli, Z. Hussain, Z.-X. Shen, Rev. Mod. Phys. 75, 473 (2003).

[54] P.C. Snijders, H.H. Weitering, Rev. Mod. Phys. 82, 307 (2010).

[55] M.Z. Hasan, C.L. Kane, Rev. Mod. Phys. 82, 3045 (2010).

[56] P. Szumniak, D. Chevallier, D. Loss, J. Klinovaja, Phys. Rev. B 96, 041401 (2017).

[57] D. Sticlet, C. Pascu Moca, B. Dóra, Phys. Rev. B 102, 075437 (2020).

[58] Supplemental Material in: Ref. [36].

[59] A.V. Balatsky, I. Vekhter, J.-X. Zhu, Rev. Mod. Phys. 78, 373 (2006).

[60] M.T. Deng, S. Vaitiekenas, E.B. Hansen, J. Danon, M. Leijnse, K. Flensberg, J. Nygård, P. Krogstrup, C.M. Marcus, Science 354, 1557 (2016).
[61] E. Prada, R. Aguado, P. San-Jose, Phys Rev. B 96, 085418 (2017).

[62] S. Hoffman, D. Chevallier, D. Loss, J. Klinovaja, Phys. Rev. B 96, 045440 (2017).

[63] E. Vernek, P.H. Penteado, A.C. Seridonio, J.C. Egues, Phys. Rev. B 89, 165314 (2014).

[64] J. Barański, A. Kobiałka, T. Domański, J. Phys. Condens. Matter 29, 075603 (2016).

[65] T. Zienkiewicz, J. Barański, G. Górski, T. Domański, J. Phys. Condens. Matter 32, 025302 (2019).

[66] Ch.-X. Liu, J.D. Sau, S. Das Sarma, Phys. Rev. B 97, 214502 (2018).

[67] L.S. Ricco, M. de Souza, M.S. Figueira, I.A. Shelykh, A.C. Seridonio, Phys. Rev. B 99, 155159 (2019).

[68] O.A. Awoga, J. Cayao, A.M. BlackSchaffer, Phys. Rev. Lett. 123, 117001 (2019).

[69] P. Zhang, F. Nori, New J. Phys. 18, 043033 (2016). 